\title{
Deep Learning Based Atom Segmentation and Noise and Missing-Wedge Reduction for Electron Tomography
}

\author{
Mengshu Ge ${ }^{1}$, and Huolin L. Xin ${ }^{1}$ \\ 1. Center for Functional Nanomaterials, Brookhaven National Laboratory, Upton, NY, USA
}

Object segmentation can be viewed as a process of disconnecting the area of interested (or foreground area) from the background. It is a popular problem in the fields of medical image processing and diagnosis, video surveillance, autonomous driving, etc. There are many conventional algorithms developed for this task, including threshold-based methods, clustering-based methods, edge detection methods, graph methods, and etc [1]. Although many of these algorithms can achieve good performance for some predefined sceneries, they tend to fail when interferences from the background are strong and unpredictable. Particularly, for atomic resolution annular dark-field scanning transmission electron microscopy (ADF-STEM), so far there is no established algorithm that is robust enough to segment all atomic columns when there is large thickness variation in a recorded image. For example, without human assistance it is always difficult to segment/locate the thinner atomic columns that are close to the edge/surface of a nanoparticle due to their weaker signals.

Recently, deep convolutional neural networks (CNNs) have achieved great performance on many visual tasks, including image recognition, image generation, object segmentation. It has been shown that given sufficient training datasets, CNNs can segment and classify objects against complicated backgrounds with high accuracy and they can even outperform humans in terms of precision and accuracy. Locating atomic columns in an ADF-STEM image similarly can be treated as an object segmentation problem. In this study, we leverage recent advances in this field and build a deep-learning Atom Segmention Net using an encoder-decoder (or U-net) architecture. The main idea of U-net is to propagate context information to higher resolution layers by concatenating high resolution features and upsampled features. U-net has been proved successful on biomedical segmentation applications such as cell tracking and segmentation [2].

In our model, U-net is mainly used for ADF-STEM image segmentation. Then Circular Hough Transform is used to detect segmented atomic column. The challenge in atom segmentation for arbitrary ADF-STEM images lies in five dominant unpredictable factors: 1) noise, 2) thickness variation and background, 3) imaging condition, 4) crystal structure and zone axis, and 5) field of view. For our network to learn how to recognize atomic columns in all different possible scenarios, we have built a library of 10,000 atomic resolution ADF-STEM images by randomly combining different crystal structure, zone axe, field of view, imaging condition, thickness variation, background pattern, and noise level. These images are synthesized by computer algorithms so we know exactly where the atomic columns are, i.e. ground truth. This synthetic approach allows us to easily scale up and augment the training library to prevent over-fitting.

Apart from 2D projection imaging, in this talk, I will discuss using CNN to reduce noise and missingedge artefacts for 3D electron tomography datasets [3].

References:

[1] Zaitoun, Nida M., and Musbah J. Aqel. Procedia Computer Science 65 (2015), p. 797. 
[2] Olaf Ronneberger, Philipp Fischer, Thomas Brox, Medical Image Computing and ComputerAssisted Intervention (MICCAI), Springer, LNCS, Vol.9351: 234-241, 2015.

[3] This research used resources of the Center for Functional Nanomaterials, which is a U.S. DOE Office of Science Facility, at Brookhaven National Laboratory under Contract No. DE-SC0012704.
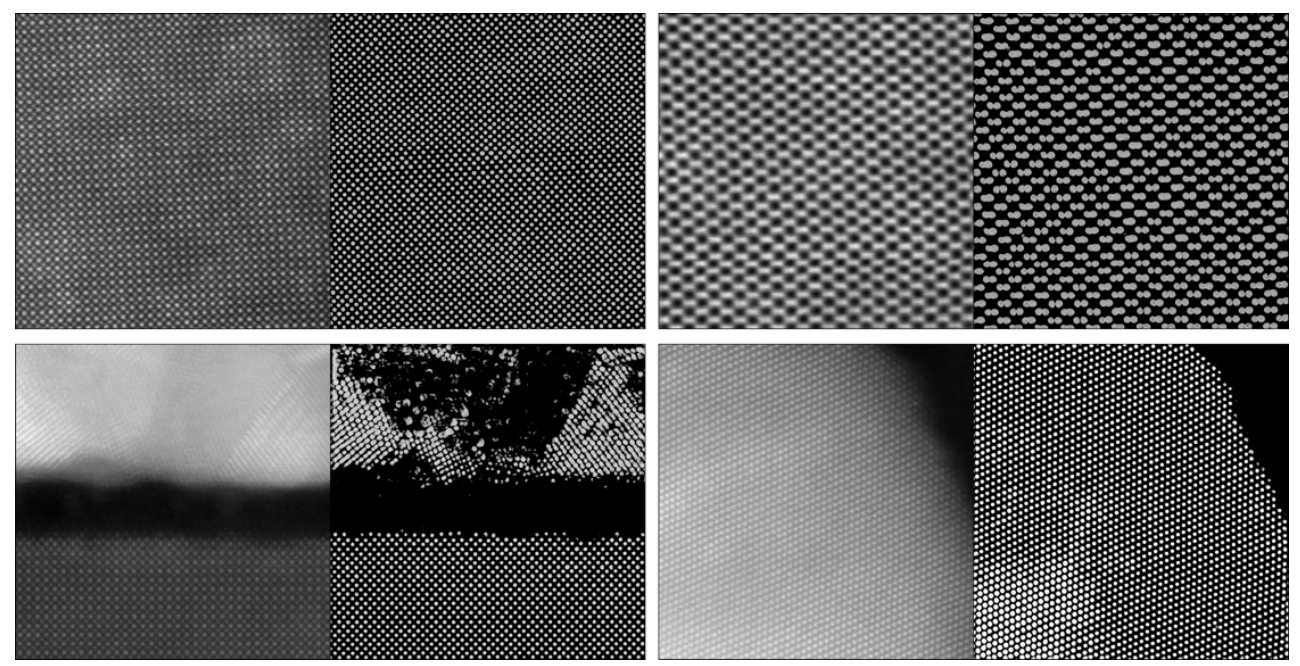

Figure 1. Application of our Atom Segmentation Net to experimental ADF-STEM images.

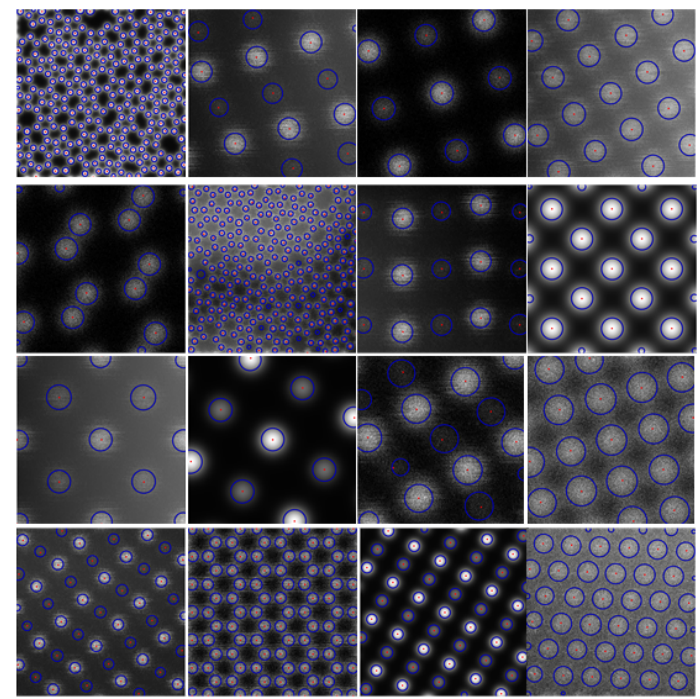

Figure 2. Atomic column localization by Atom Segmentation Net in conjunction with Circular Hough Transform.

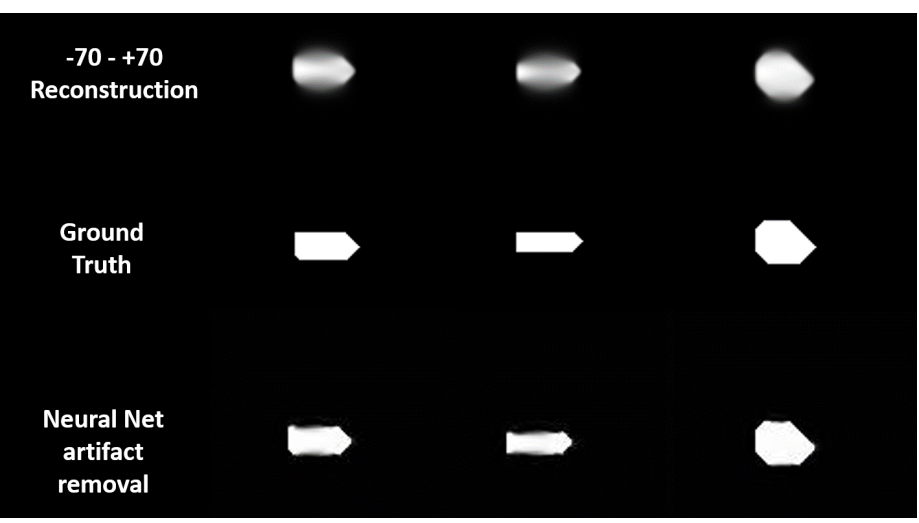

Figure 3. Using a residual analysis neural network to remove missing wedge artifacts. 